\title{
EL CONECTAMEF Y SU INCIDENCIA EN LA CALIDAD DEL GASTO PÚBLICO Y AHORRO DE LAS MUNICIPALIDADES DE PUNO, PERÚ
}

\author{
Félix Henry GUTIÉRREZ CASTILLO ${ }^{1}$ \\ Ronny Alexander GUTIÉRREZ CASTILLO ${ }^{2}$ \\ Félix Alejandro GUTIÉRREZ GALLEGOS ${ }^{3}$ \\ Ana María RAMOS RAMOS ${ }^{4}$ \\ Sergio Paúl GUTIÉRREZ CASTILLO ${ }^{5}$
}

\begin{tabular}{|lcc|}
\hline Recibido & $:$ & 30.03 .2020 \\
Aceptado & $:$ & 06.06 .2020 \\
Publicado & $:$ & 06.07 .2020 \\
\hline
\end{tabular}

RESUMEN: El objetivo de la investigación fue determinar si el nivel de cumplimiento de los servicios brindados por el Centro de Atención al Usuario del Ministerio de Economía de Finanzas CONECTAMEF tuvo una incidencia significativa en la calidad del gasto público de las municipalidades de la región Puno; periodo 2017. El estudio se realizó bajo un enfoque cuantitativo, siendo una investigación no experimental, con un diseño de investigación transeccional, de alcance descriptivo y correlacional; la población estuvo conformada por las 96 municipalidades distritales, se utilizó el muestreo censal por ser tener una población reducida, tomando todos los elementos para el estudio; como técnicas de investigación se utilizó el análisis documental y la encuesta, el instrumento utilizado fue el cuestionario. Utilizando la prueba estadística Chi-Cuadrado " $\mathrm{x}$ " " se obtuvo como resultado un $\mathrm{p}$ $=0.001$, demostrando que, si existe relación entre las variables estudiadas, y según coeficiente de Cramer, ésta es de un $83.7 \%$; concluyendo que existe una incidencia significativa entre el cumplimiento de los servicios brindados por el CONECTAMEF y el nivel de la calidad del gasto público de las municipalidades.

Palabras claves: Calidad, gasto, incidencia, municipalidades y servicio.

\section{The CONECTAMEF and its incidence in the quality of public expense and saving of Puno's Municipalites - Perú}

\begin{abstract}
The purpose of the investigation was to determine whether the level of compliance with the services provided by the Centro de Atención al Usuario del Ministerio de Economía de Finanzas CONECTAMEF had a significant impact on the public spending quality by the municipalities of Puno region; period 2017. The study was carried out under a quantitative approach, being a non-experimental investigation, with a cross-sectional research design, of descriptive and correlational scope; the population was made up of the 96 district municipalities, because the study had a small population the census sampling was used, taking all the elements for the study; documentary analysis and the survey were used as research techniques, the instrument used was the questionnaire. Using the Chi-square statistical test " $\mathrm{x}$ ", a $\mathrm{p}=0.001$ was obtained as a result, demonstrating that there is a relationship between the variables studied, and according to the Cramer coefficient, it is $83.7 \%$; concluding that exist a significant incidence between the compliance of the services provided by CONECTAMEF and the quality's level of the public spending of the municipalities.
\end{abstract}

Keywords: Quality, expense, incidence, municipalities and service.

\footnotetext{
${ }^{1}$ Universidad Nacional del Altiplano.

${ }^{2}$ Universidad Nacional del Altiplano. Email: roca_227@ hotmail.com

${ }^{3}$ Universidad Nacional del Altiplano.

${ }^{4}$ Universidad Nacional del Altiplano.

${ }^{5}$ Universidad Nacional del Altiplano
} 


\section{Journal of the Academy $|56|$}

\section{INTRODUCCIÓN}

En los últimos años la economía peruana ha crecido significativamente sin embargo este crecimiento no ha sido bien acompañado por un adecuado nivel de calidad del gasto público en muchas regiones y no se ha podido satisfacer las necesidades ni lograr mejoras en las condiciones de vida de la población. Es por ello que actualmente en las entidades públicas se habla mucho de la importancia de mejorar el nivel de calidad del gasto público, mediante la ejecución de proyectos y programas sociales que constituye una pieza clave para el desarrollo de nuestro país (Gutiérrez, 2018).

En relación a la importancia de este tema, el Ministerio de Economía y Finanzas - MEF, siendo el organismo del Poder Ejecutivo, encargado de planear, dirigir y controlar los asuntos relativos a presupuesto, tesorería, endeudamiento, contabilidad, política fiscal, inversión pública y política económica social; crea los Centros de Servicios de Atención al Usuario - CONECTAMEF, siendo estos quienes brindan los servicios de atención de consultas, asistencia técnica, capacitación y mesa de partes, a funcionarios de las entidades públicas. Actualmente se cuenta con 27 centros de servicios ubicados estratégicamente en la costa, sierra y selva del Perú, su objetivo principal es consolidarse como centros integrales de acompañamiento técnico especializado en las regiones, ofreciendo una atención personalizada, directa y de fácil acceso de los servicios de competencia exclusiva del Ministerio de Economía y Finanzas (MEF, 2018).

Respecto a los antecedentes referidos al tema de investigación tenemos los estudios de: Vilca (2015) en su investigación acerca de los factores que inciden en el desempeño de los gastos de capital en los gobiernos locales de la región de Puno 2013, concluyó que las municipalidades con restricciones de capacidad técnica vinculada a inversiones, administración, contabilidad y finanzas afectan de manera negativa el desempeño de gastos de capital con una restricción de la inversión en menos de 0.18; Cacsire (2012) en su investigación sobre los efectos del proceso de descentralización administrativa y presupuestal en la gestión de las direcciones regionales del gobierno regional Puno - Caso DIRCETUR, concluyó que los indicadores de eficacia, respecto al Presupuesto Institucional de Apertura, presenta un valor promedio de una ejecución del gasto anual de 0.93 y respecto al Presupuesto Institucional Modificado, la ejecución del gasto anual presenta un valor de 0.96, siendo ambos indicadores menor que 1 , lo que caracteriza un indicador deficiente.

Asimismo en otras investigaciones se analizó el impacto de muchos proyectos y programas sociales como: Catacora (2017), quien evaluó el impacto de un proyecto, en el cual concluyó que este ha sido beneficioso para para sus beneficiarios; Apaza (2014) que investigó el impacto del programa JUNTOS concluyó que la aplicación exitosa de la utilización del programa en inversión de largo plazo (en educación y salud) parece haber dejado sin variación los beneficios marginales esperados de tener préstamos en el corto plazo.

Por su parte, Espezúa (2014) investigó el tema de la política social y seguridad alimentaria en Puno caso: El Programa del Vaso de Leche, donde concluyó que la dinámica de organización y funcionamiento de los programas sociales alimentarios, da un aporte significativo a los grupos más vulnerables de la ciudad de Puno, al producir efectos socioeconómicos positivos en cuanto ataca la inseguridad alimentarias, refuerza la organización municipal, y fortalece las organizaciones de base: la política alimentaria estatal mejora las condiciones de vida de la población, da apoyo directo a los pobres extremos, tiende a superar la inseguridad alimentaria, así como capacita a las socias para un mejor funcionamiento del mismo.

Maquera (2009) en su investigación del análisis de la descentralización de los programas sociales en Ilave, con énfasis en los comedores populares, concluyó que el desarrollo local es entendido como el proceso mediante el cual se dinamiza las potencialidades humanas, físicas, culturales, recursos naturales y empresariales en un contexto social con la finalidad de mejorar las condiciones sociales y económicas de los beneficiarios; finalmente Choquehuanca (2007) quien en su investigación sobre la valoración de

Como citar: Gutiérrez Castillo, Félix Henry; Gutiérrez Castillo, Ronny Alexander; Gutiérrez Gallegos, Félix Alejandro; 


\section{Journal of the Academy | $57 \mid$}

beneficios para proyectos de drenaje de aguas pluviales; caso: ciudad de Juliaca; concluyó que los beneficios identificados por el proyecto con la metodología para estimar beneficios de valoración contingente son: habitantes de las viviendas con ahorro de costos de operación y mantenimiento de vehículos de transporte, ahorro en costos de desplazamiento y tiempo.

Por otra parte, es necesario definir también el término "Servicio", que según Muller (2003) indicó que un servicio es una acción utilitaria que satisface una necesidad específica de un cliente. Asimismo, según Zuleta et al., (2014) sostienen que los servicios son acciones, procesos y ejecuciones intangibles; por su parte Serna (1999) afirma que el servicio al cliente es el conjunto de estrategias que una compañía diseña por satisfacer mejor que sus competidores, las necesidades y expectativas de sus clientes externos. Olvera et al., (2009) señalaron que el servicio es una actividad humana que se caracteriza por ofrecer tu tiempo, habilidades y conocimientos en beneficio de los demás.

En ese sentido, dentro de los servicios que brindan los CONECTAMEF están: Atención de consultas; asistencia técnica; capacitación y mesa de partes (MEF, 2018). Asimismo, las temáticas atendidas por los especialistas de los Sistemas Administrativos son las siguientes: Presupuesto Público; Contabilidad Pública; Endeudamiento y Tesoro Público; Inversión Pública; Tecnologías de Información y Recursos Públicos.

Asimismo, es de precisar que el término "Calidad" es un concepto muy subjetivo y no existe una definición exacta ni estandarizada sobre lo que es la calidad como tal cada persona de acuerdo a las expectativas del servicio que espera recibir la percibe de diferente manera. Al respecto, Cantú (1997) indica que las normas ISO 9000 expresan el término calidad como la integración de las características que determinan en qué grado un producto satisface las necesidades de su consumidor; sin embargo, Duque (2005) precisa que la calidad se refiere únicamente al valor que un consumidor le otorga a un producto o servicio, entendiendo con esto que el cliente es quien determinará la calidad del producto y/o servicio que se le otorga.

Por su parte Olvera et al., (2009) señalaron que la calidad es el grado de satisfacción que obtiene el cliente en relación con su expectativa inicial, es un aspecto totalmente subjetivo y personal, sin embargo, se debe buscar la forma de lograr la superación de esta satisfacción, no solo cubrir una necesidad, sino ofrecer más.

Respecto al Gasto Público, se debe precisar que este el conjunto de erogaciones que por concepto de gastos corrientes, gastos de capital y servicio de deuda, realizan las entidades con cargo a los créditos presupuestarios respectivos, para ser orientados a la atención de la prestación de los servicios públicos y acciones desarrolladas de conformidad con las funciones y objetivos institucionales (MEF, 2018).

Ahora, debemos entender que las Municipalidades son los Órganos de gobierno local que se ejerce en las circunscripciones provinciales y distritales de cada una de las regiones del país, con las atribuciones, competencias y funciones que le asigna la Constitución Política del Perú, la Ley Orgánica de Municipalidades y la Ley de Bases de la Descentralización. En la capital de la república el gobierno local lo ejerce la Municipalidad Metropolitana de Lima. En los centros poblados funcionan municipalidades conforme a ley (Ministerio de Comercio Exterior y Turismo, 2002).

Se tiene tres tipos de municipalidades como son: las Municipalidades provinciales que ejercen el gobierno local en las demarcaciones provinciales; las Municipalidades distritales que ejercen el gobierno local en las demarcaciones distritales y las Municipalidades de centros poblados que se crean por ordenanza municipal provincial y ejercen funciones delegadas, las que se establecen en la ordenanza que las crea. Para el cumplimiento de sus funciones las municipalidades provinciales y distritales deben asignarles recursos económicos de manera mensual (Ministerio de Comercio Exterior y Turismo, 2002).

En ese sentido, el objetivo planteado de la investigación fue: Determinar si el nivel de cumplimiento de los servicios brindados por el CONECTAMEF tuvo una incidencia significativa en la calidad del gasto

Como citar: Gutiérrez Castillo, Félix Henry; Gutiérrez Castillo, Ronny Alexander; Gutiérrez Gallegos, Félix Alejandro;

Ramos Ramos, Ana María y Gutiérrez Castillo, Sergio Paúl (2020). El CONECTAMEF y su incidencia en la calidad del gasto público y ahorro de las Municipalidades de Puno, Perú. Journal of Academy núm 3, 55-66. www.doi.org/10.47058/joa3.5 


\section{Journal of the Academy $|58|$}

público y el ahorro de las municipalidades de la región de Puno, durante el periodo 2017, para lo cual se analizó y evaluó si el nivel de cumplimiento de los servicios brindados por el CONECTAMEF, tiene incidencia significativa con el nivel de calidad del gasto público y con el ahorro anual de las municipalidades distritales de la región de Puno.

\section{DESARROLLO}

\section{Materiales y Métodos Lugar de estudio}

El trabajo de investigación se desarrolló en el ámbito de cobertura que tiene el CONECTAMEF en la región de Puno que esta subdividida en 13 provincias y 109 distritos; región que se encuentra ubicada al extremo sur este del Perú, entre los $13^{\circ} 00^{\prime} 00^{\prime \prime}$ y $17^{\circ} 17^{\prime} 30^{\prime \prime}$ de latitud sur y los $71^{\circ} 06^{\prime} 57^{\prime \prime}$ y $68^{\circ} 48^{\prime} 46^{\prime \prime}$ de longitud oeste del meridiano de Greenwich; cuenta con una extensión territorial de $71999,00 \mathrm{~km}^{2}$ (6\% del territorio nacional) siendo el quinto departamento más grande en el ámbito nacional. Limita por el norte con la región de Madre de Dios, por el este con la República de Bolivia, por el sur con la región de Tacna y la República de Bolivia y por el oeste con las regiones de Moquegua, Arequipa y Cusco.

\section{Descripción detallada por objetivos específicos}

Para el cumplimiento de los objetivos del trabajo de investigación se utilizó el tipo de investigación no experimental con un diseño de investigación transeccional de análisis descriptivo - correlacional.

El alcance de la investigación, para determinar la incidencia que tiene el nivel de calidad del gasto público, se enmarca en el tipo correlacional, donde la población estuvo conformada por las 96 municipalidades distritales de la región Puno, y en este caso debido a que los sujetos de la población constituyen un grupo reducido se optó por una muestra de tipo no probabilística - muestreo censal, donde se considera a la totalidad de la población.

Ahora, para determinar el procedimiento de la metodología empleada para la recolección de datos debemos tener en cuenta que el CONECTAMEF Puno, brinda los siguientes servicios: atención de consultas, asistencia técnica, mesa de partes y capacitación en los diferentes sistemas administrativos de su competencia, como son: Presupuesto público, contabilidad pública, endeudamiento y tesoro público, inversión pública, gestión de recursos públicos y a través de la oficina general de tecnologías de la información (OGTI) brinda el servicio de soporte en los aplicativos informáticos SIAF y SIGA; para ello cuenta con especialistas en cada tema.

En la investigación se analizó el cumplimiento de cada servicio por cada municipalidad distrital de la región Puno; los datos fueron recolectados de la base de datos del CONECTAMEF; data en la que se registra el tipo de servicio que se prestó a cada entidad; esta información fue concordada y se vertió en una matriz, en la cual, para conocer los niveles de cumplimiento de los servicios, se calificó utilizando la escala de Likert de 5 puntos, desde 1 (Muy Insatisfactorio) hasta 5 (Muy Satisfactorio), esto para cada servicio prestado en cada tema; para que finalmente mediante promedios ponderados se obtenga el puntaje final de las municipalidades distritales agrupadas en las 13 provincias de la región Puno.

Luego se analizó el gasto realizado por cada municipalidad distrital de la región Puno, con esto se pudo identificar a las municipalidades que deben ser atendidas con mayor incidencia, teniendo como criterio que mientras más alto sea el porcentaje de los programas presupuestales respecto a las asignaciones presupuestales que no resultan en productos, mayor será el nivel de calidad del gasto público de la municipalidad distrital analizada, considerando para ello puntajes de: 1 (Bajo) hasta 4 (Muy Bueno); teniendo los puntajes respectivos, se sacó el promedio de estos para obtener el puntaje final de las municipalidades distritales agrupadas en las 13 provincias de la región de Puno. 


\section{Journal of the Academy | $59 \mid$}

Seguidamente para poder determinar el ahorro anual de las municipalidades, contemplado en nuestro objetivo específico 2, se utilizó la estadística descriptiva, tomando en cuenta para ello las áreas que intervienen para realizar las gestiones respectivas que exige cada sistema administrativo en la gestión pública que realiza cada entidad.

Finalmente, para el procesamiento y análisis de datos, para cada objetivo específico planteado en el trabajo de investigación, se tuvo en cuenta la evaluación y análisis de los dos instrumentos utilizados, la información que fue consolidada en tablas utilizando el siguiente procedimiento: Tabulación de datos; descripción, interpretación y análisis de datos, para obtener la representación escrita, tabular y gráfica; se aplicó porcentajes, estadísticas y esquemas básicos, utilizando la estadística descriptiva; finalmente se explicó los resultados de la investigación, utilizando el Software IBM-SPSS versión 22.

Asimismo, precisar que para determinar la relación entre las variables de la investigación se utilizó la prueba no paramétrica: Coeficiente de Correlación de Pearson (Chi cuadrado) que, es una prueba estadística para analizar la relación entre dos variables medidas en un nivel por intervalos o de razón (Hernández et al., 2010).

\section{Resultados y Discusión}

Determinación del nivel de cumplimiento de los servicios brindados por el CONECTAMEF y su incidencia en el nivel de calidad del gasto público de las municipalidades distritales de la región de Puno.

Respecto a los niveles de calidad del gasto público de las municipalidades distritales de la región de Puno, tomando en cuenta el portal web "Consulta Amigable" del Ministerio de Economía y Finanzas, que muestra información actualizada por cada periodo que se requiera; para el trabajo de investigación se recolecto la información desde el periodo 2012 (año en que el CONECTAMEF brinda servicios en Puno), se consideró el gasto realizado por cada municipalidad, por categorías presupuestarias: programas presupuestales y asignaciones presupuestales que no resultan en productos; dicha información si la vemos de forma general observamos un comportamiento óptimo, tal y conforme se muestra en la figura 1.

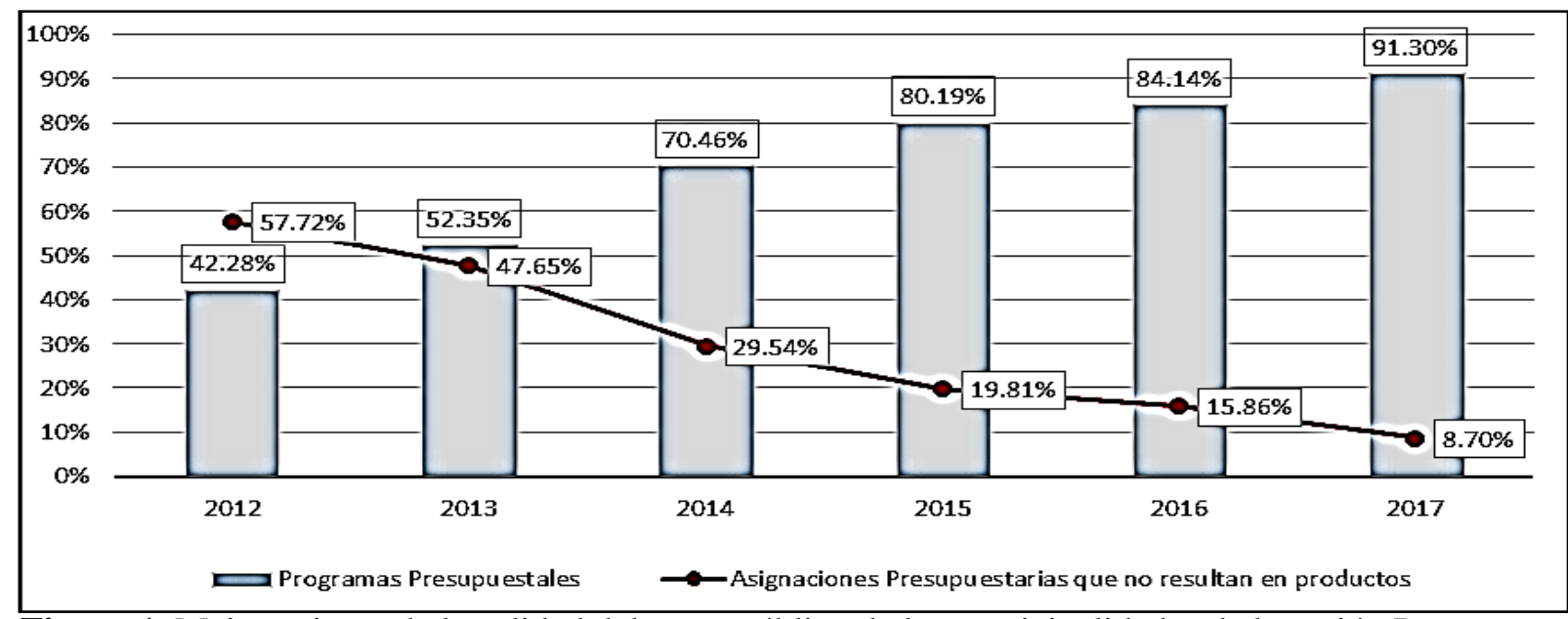

Figura 1. Mejoramiento de la calidad del gasto público de las municipalidades de la región Puno.

Fuente: Datos obtenidos del portal web "Consulta Amigable".

Ahora, para obtener los resultados del objetivo específico 1, que refiere al cumplimiento de los servicios brindados del CONEECTAMEF y su incidencia en el nivel de calidad de gasto púbico; y 


\section{Journal of the Academy $|60|$}

tomando en cuenta la metodología empleada; detallada anteriormente; se obtuvo los siguientes datos (tablas 1 y 2)

Tabla 1.

Cumplimiento de los servicios brindados por el CONECTAMEF Puno.

\begin{tabular}{lcccc}
\hline Cumplimiento de los servicios & Fi & hi\% & Fi & Hi\% \\
\hline Muy Insatisfactorio & 0 & 0.00 & 0 & 0.00 \\
Insatisfactorio & 0 & 0.00 & 0 & 0.00 \\
Indiferente & 4 & 30.77 & 4 & 30.77 \\
Satisfactorio & 8 & 61.54 & 12 & 92.31 \\
Muy Satisfactorio & 1 & 7.69 & 13 & 100.00 \\
Total & $\mathbf{1 3}$ & $\mathbf{1 0 0 . 0 0}$ & &
\end{tabular}

Fuente: Base de datos del CONECTAMEF Puno; periodo 2017.

Tabla 2.

Nivel de calidad del gasto público de las municipalidades distritales de la región Puno.

\begin{tabular}{lcc}
\hline Calidad de gasto público & fi & hi\% \\
\hline Bajo & 0 & 0.00 \\
\hline Mínimo Necesario & 2 & 15.39 \\
Bueno & 10 & 76.92 \\
Muy Bueno & 1 & 7.69 \\
Total & $\mathbf{1 3}$ & $\mathbf{1 0 0 . 0 0}$ \\
\hline
\end{tabular}

Fuente: Consulta Amigable MEF - 2017.

En base a los resultados reflejados en las tablas 1 y 2 se elaboró la tabla 3, que representa la relación entre el nivel de cumplimiento de los servicios brindados por el CONECTAMEF y la calidad del gasto público de las municipalidades distritales

Tabla 3.

Cumplimiento de los servicios del CONECTAMEF y nivel de calidad del gasto público de las municipalidades distritales de la región Puno.

\begin{tabular}{|c|c|c|c|c|c|c|c|c|c|c|}
\hline \multirow{2}{*}{$\begin{array}{c}\text { Nivel calidad del gasto } \\
\text { público } \\
\text { Cumplimiento de los } \\
\text { Servicios }\end{array}$} & \multicolumn{2}{|c|}{ Bajo } & \multicolumn{2}{|c|}{$\begin{array}{c}\text { Mínimo } \\
\text { Necesario }\end{array}$} & \multicolumn{2}{|c|}{ Bueno } & \multicolumn{2}{|c|}{ Muy Bueno } & \multicolumn{2}{|c|}{ Total } \\
\hline & $\mathbf{f}_{\mathbf{i}}$ & $\%$ & $\mathbf{f}_{\mathbf{i}}$ & $\%$ & $\mathbf{f}_{\mathbf{i}}$ & $\%$ & $\mathbf{f}_{\mathbf{i}}$ & $\%$ & $\mathbf{f}_{\mathbf{i}}$ & $\%$ \\
\hline Muy Insatisfactorio & 0 & 0.00 & 0 & 0.00 & 0 & 0.00 & 0 & 0.00 & 0 & 0.00 \\
\hline Insatisfactorio & 0 & 0.00 & 0 & 0.00 & 0 & 0.00 & 0 & 0.00 & $\mathbf{0}$ & 0.00 \\
\hline Indiferente & 0 & 0.00 & 2 & 15.39 & 2 & 15.39 & 0 & 0.00 & 4 & 30.77 \\
\hline Satisfactorio & 0 & 0.00 & 0 & 0.00 & 8 & 61.54 & 0 & 0.00 & 8 & 61.54 \\
\hline Muy Satisfactorio & 0 & 0.00 & 0 & 0.00 & 0 & 0.00 & 1 & 7.69 & 1 & 7.69 \\
\hline Total & $\mathbf{0}$ & 0.00 & 2 & 15.39 & 10 & 76.92 & 1 & 7.69 & 13 & 100.00 \\
\hline
\end{tabular}

Fuente: Tablas de recolección de datos, resultados reflejados en las tablas 1 y 2.

La contrastación de hipótesis con el estadístico Chi-Cuadrado " $\mathrm{X}^{2}$ ", a un nivel de significancia de 5\% $=0.05$ (95\% de nivel de confianza); arrojo como resultado un valor de $\mathrm{p}=0,001 \mathrm{y}$ siendo este menor a 0.05 se aceptó la hipótesis alterna que indica: que existe una relación significativa entre el nivel de cumplimiento de los servicios brindados por el CONECTAMEF y el nivel de calidad del gasto público 


\section{Journal of the Academy $|61|$}

de las municipalidades distritales de la región Puno; y tiene un grado de relación según el coeficiente V de Cramer de 83.7\%; quedando de esta manera contrastada la hipótesis.

Ahora, respecto a los antecedentes de investigación mencionados, tenemos varios de ellos que hablan sobre dimensiones o atributos de calidad que están muy relacionados con la inversión pública (Gutiérrez, 2018) y que se complementan con los de este trabajo de investigación, en ese sentido se mencionan los siguientes:

De los resultados obtenidos en el trabajo de investigación, se identifica claramente que los servicios que brinda el CONECTAMEF Puno (MEF, 2018), tienen una incidencia significativa en la calidad del gasto público de las municipalidades distritales de la región Puno, hecho que coincide con los resultados de Vilca (2015), quien en su investigación sobre los: Factores que inciden en el desempeño de los Gastos de Capital en los Gobiernos Locales de la Región de Puno, 2013 concluyó que, las municipalidades con restricciones de capacidad técnica vinculada a inversiones, administración, contabilidad y finanzas afectan de manera negativa el desempeño de gastos de capital con una restricción de la inversión en menos de 0.18 .

Asimismo, Catacora (2017); Apaza (2014); Espezúa (2014); Maquera (2009) y Choquehuanca (2007), quienes desarrollaron temas respecto a la evaluación de proyectos y programas sociales, llegan a la misma conclusión del trabajo de investigación, resaltando que el buen manejo de estos, darán una incidencia positiva en la gestión pública.

\section{Determinación del nivel de cumplimiento de los servicios brindados por el CONECTAMEF y su incidencia en el ahorro anual de las municipalidades distritales de la región de Puno.}

Según la metodología utilizada para la recolección de datos para este objetivo, se tiene los siguientes datos, relacionados a las actividades que realizaban antes las direcciones del Ministerio de Economía y Finanzas:

\section{Dirección General de Contabilidad Pública - DGCP}

Actividad 1: Información para la Cuenta General de la República.

Actividad 2: Conciliación de saldos de fondos públicos - SAFOP.

Actividad 3: Conciliación del marco legal del presupuesto.

\section{Dirección General de Presupuesto Público - DGPP}

Actividad 1: Formulación y presentación del presupuesto institucional de apertura.

Actividad 2: Presentación y sustentación de la evaluación del marco presupuestal.

Dirección General de Endeudamiento y Tesoro Público - DGETP

Actividad 1: Modificación del registro de responsables del manejo de cuentas.

\section{Dirección General de Inversión Pública - DGIP}

Actividad 1: Presentación y recepción de expedientes concurso FONIPREL

Estas actividades actualmente ya se dejaron de realizar, debido a que desde la implementación del CONECTAMEF los funcionarios de las entidades públicas ya no tienen la necesidad de viajar a la capital para realizar dichos tramites; siendo esta entidad descentralizada el nexo directo con el Ministerio de Economía y Finanzas.

Ahora, para calcular el ahorro de las municipalidades, se tomaron en cuenta los siguientes criterios: Numero de comisionados, días de comisión, monto asignado de viáticos y pasajes (el monto promedio de viáticos S/ 320.00 por día y S/ 250.00 por concepto de pasajes) y cuántas veces al año se realizaba la actividad.

Por ejemplo, en la actividad 1 ante la Dirección General de Contabilidad Pública - DGCP, el ahorro se detalla en la tabla 4. 


\section{Journal of the Academy $|62|$}

Tabla 4.

Cumplimiento de los servicios del CONECTAMEF y nivel de calidad del gasto público de las municipalidades distritales de la región Puno, periodo 2017.

\begin{tabular}{lcccc}
\hline Comisionados & $\begin{array}{c}\text { Días } \\
\text { Comisión }\end{array}$ & $\begin{array}{c}\text { Viáticos } \\
\text { S/ }\end{array}$ & $\begin{array}{c}\text { Pasajes } \\
\text { S/ }\end{array}$ & $\begin{array}{c}\text { Total en } \\
\text { Soles }\end{array}$ \\
\hline Alcalde / Gerente & 3 & 960.00 & 250.00 & $1,210.00$ \\
Contador & 3 & 960.00 & 250.00 & $1,210.00$ \\
Presupuesto & 3 & 960.00 & 250.00 & $1,210.00$ \\
Tesorero & 3 & 960.00 & 250.00 & $1,210.00$ \\
& Gasto por municipalidad & & $\mathbf{4 , 8 4 0 . 0 0}$
\end{tabular}

Fuente: Datos obtenidos de los funcionarios.

Actualmente, el especialista en Contabilidad Pública del CONECTAMEF, recibe la carpeta de la rendición de cuentas; valida la información entre el SIAF y SICON, registra en el sistema de trámite de la DGCP y firma el acta de recepción de la información del contenido de carpetas. Con esto las municipalidades en conjunto hacen un ahorro en gastos de viáticos y pasajes, el cual se detalla en la tabla 5 .

Tabla 5.

Ahorro a las municipalidades de la región. Actividad 1 (DGCP)

\begin{tabular}{lccc}
\hline Periodo & $\begin{array}{c}\mathbf{N}^{\circ} \text { de } \\
\text { Municipalidades }\end{array}$ & $\begin{array}{c}\text { Ahorro por } \\
\text { cada municipalidad }\end{array}$ & $\begin{array}{c}\text { Ahorro en } \\
\text { Soles }\end{array}$ \\
\hline Al I Trimestre & 109 & $4,840.00$ & $527,560.00$ \\
\hline Al II Trimestre & 109 & $4,840.00$ & $527,560.00$ \\
\hline Al III Trimestre & 109 & $4,840.00$ & $527,560.00$ \\
Al IV Trimestre & 109 & $4,840.00$ & $527,560.00$ \\
& Total ahorro & & $\mathbf{2 , 1 1 0 , 2 4 0 . 0 0}$ \\
\hline
\end{tabular}

Respecto a la actividad 2, ante la DGCP “Conciliación de Saldos de Fondos Públicos SAFOP”, antes se tenía el siguiente gasto que realizaba cada municipalidad.

Tabla 6.

Gasto estimado que asumía cada municipalidad para la Actividad 2: Conciliación de Saldos de Fondos Públicos SAFOP

\begin{tabular}{|ccccc}
\hline Comisionados & $\begin{array}{c}\text { Días } \\
\text { Comisión }\end{array}$ & $\begin{array}{c}\text { Viáticos } \\
\text { S/ }\end{array}$ & $\begin{array}{c}\text { Pasajes } \\
\text { S/ }\end{array}$ & Total en Soles \\
\hline Contador & 2 & 640.00 & 250.00 & 890.00 \\
Tesorero & 2 & 640.00 & 250.00 & 890.00 \\
& Gasto por municipalidad & & $\mathbf{1 , 7 8 0 . 0 0}$ \\
\hline
\end{tabular}

Fuente: Datos obtenidos de los funcionarios.

Ahora, el especialista en Contabilidad Pública del CONECTAMEF: Recibe la carpeta de los Saldos de Fondos Públicos; valida la información con los estados financieros, registra en el sistema de trámite de la DGCP y firma el acta de recepción del Saldo de Fondos Públicos. Con esto las municipalidades en conjunto hacen un ahorro en gasto de viáticos y pasajes, el cual se detalla a continuación en la tabla 7. 


\section{Journal of the Academy $|63|$}

Tabla 7.

Ahorro a las municipalidades de la región. Actividad 2 (DGCP)

\begin{tabular}{cccc}
\hline Periodo & $\begin{array}{c}\mathbf{N}^{\circ} \text { de } \\
\text { Municipalidades }\end{array}$ & $\begin{array}{c}\text { Ahorro por } \\
\text { cada municipalidad }\end{array}$ & Ahorro en Soles \\
\hline Anual & 109 & $1,780.00$ & $194,020.00$ \\
& Total ahorro & & $\mathbf{1 9 4 , 0 2 0 . 0 0}$ \\
\hline
\end{tabular}

En relación a la actividad 3, ante la DGCP "Conciliación del marco legal del presupuesto", antes se tenía el siguiente gasto que realizaba cada municipalidad.

Tabla 8.

Gasto estimado que asumía cada municipalidad para la Actividad 3: Conciliación del marco legal del presupuesto

\begin{tabular}{ccccc}
\hline \multirow{2}{*}{ Comisionados } & \multirow{2}{*}{ Días Comisión } & $\begin{array}{c}\text { Viáticos } \\
\text { S/ }\end{array}$ & $\begin{array}{c}\text { Pasajes } \\
\text { S/ }\end{array}$ & Total en Soles \\
\hline Contador & 2 & 640.00 & 250.00 & 890.00 \\
Presupuesto & 2 & 640.00 & 250.00 & 890.00 \\
& Gasto por municipalidad & & $\mathbf{1 , 7 8 0 . 0 0}$ \\
\hline
\end{tabular}

Fuente: Datos obtenidos de los funcionarios.

Ahora, el especialista en Contabilidad Pública del CONECTAMEF: Recibe la carpeta de la conciliación de presupuesto por toda fuente de financiamiento, valida la información con los datos que cuenta la DGCP, registra en el sistema de trámite de la DGCP y firma el acta de conciliación. Con esto las municipalidades en conjunto hacen un ahorro en gasto de viáticos y pasajes, el cual se detalla a continuación en la tabla 9.

Tabla 9.

Ahorro a las municipalidades de la región. Actividad 3 (DGCP)

\begin{tabular}{cccc}
\hline Periodo & $\begin{array}{c}\mathbf{N}^{\circ} \text { de } \\
\text { Municipalidades }\end{array}$ & $\begin{array}{c}\text { Ahorro por } \\
\text { cada municipalidad }\end{array}$ & Ahorro en Soles \\
\hline Anual & 109 & $1,780.00$ & $194,020.00$ \\
& Total ahorro & & $\mathbf{1 9 4 , 0 2 0 . 0 0}$ \\
\hline
\end{tabular}

En resumen, las 3 actividades ante la DGCP, ahora se realizan en el CONECTAMEF, y se puede observar el ahorro generado en la tabla 10.

Tabla 10.

Resumen del ahorro generado en las tres actividades ante la Dirección General de Contabilidad Pública.

\begin{tabular}{lc}
\hline \multicolumn{1}{c}{ Actividad } & Ahorro en Soles \\
\hline Información para la Cuenta General de República & $2,110,240.00$ \\
Conciliación de Saldos de Fondos Públicos - SAFOP & $194,020.00$ \\
Conciliación del Marco Legal del Presupuesto & $194,020.00$ \\
\hline \multicolumn{1}{c}{ Total ahorro } & $\mathbf{2 , 4 9 8 , 2 8 0 . 0 0}$
\end{tabular}

De manera similar se obtuvieron los resultados para las actividades ante las demás Direcciones Generales del MEF (DGPP, DGETP y DGIP) y los resultados se muestran a continuación en la tabla 11 


\section{Journal of the Academy | $64 \mid$}

Tabla 11.

Ahorro anual de las municipalidades de la región de Puno.

Tramite realizados en los Sistemas Administrativos

Dirección General de Contabilidad Pública

Dirección General de Presupuesto Público

Dirección General de Endeudamiento y Tesoro Público

Dirección General de Inversión Pública
Ahorro en Soles

$2,498,280.00$

$1,067,110.00$

$388,040.00$

$89,000.00$

$\mathbf{4 , 0 4 2 , 4 3 0 . 0 0}$

En la tabla 12 se muestra como referencia el presupuesto anual ejecutado para la operatividad del CONECTAMEF Puno.

Tabla 12.

Presupuesto anual ejecutado del CONECTAMEF Puno.

\begin{tabular}{|l|l|l|l|l|}
\hline Cargo & Sueldo & Anual & $\begin{array}{l}\text { Viáticos y } \\
\text { caja chica }\end{array}$ & $\begin{array}{l}\text { Contratos } \\
\text { (Limpieza, } \\
\text { vigilancia y } \\
\text { alquiler de } \\
\text { local) }\end{array}$ \\
\hline Gestor de Centro & $8,000.00$ & $96,000.00$ & & $12,800.00$ \\
\hline $\begin{array}{l}\text { Asistentes de Gestión } \\
\text { de Servicios }\end{array}$ & $3,000.00$ & $36,000.00$ & & $12,800.00$ \\
\hline $\begin{array}{l}\text { Especialista en } \\
\text { Contabilidad Pública }\end{array}$ & $5,000.00$ & $60,000.00$ & & $12,800.00$ \\
\hline $\begin{array}{l}\text { Especialista en } \\
\text { Contabilidad Pública }\end{array}$ & $5,000.00$ & $60,000.00$ & & $12,800.00$ \\
\hline $\begin{array}{l}\text { Especialista en } \\
\text { Presupuesto Público }\end{array}$ & $5,000.00$ & $60,000.00$ & & $12,800.00$ \\
\hline $\begin{array}{l}\text { Especialista en } \\
\text { Presupuesto Público }\end{array}$ & $5,000.00$ & $60,000.00$ & & $12,800.00$ \\
\hline $\begin{array}{l}\text { Especialista en Endeudamiento y } \\
\text { Tesoro Público }\end{array}$ & $5,000.00$ & $60,000.00$ & & $12,800.00$ \\
\hline $\begin{array}{l}\text { Especialista en Endeudamiento y } \\
\text { Tesoro Público }\end{array}$ & $5,000.00$ & $60,000.00$ & & $12,800.00$ \\
\hline $\begin{array}{l}\text { Especialista en } \\
\text { Inversión Pública }\end{array}$ & $5,000.00$ & $60,000.00$ & & $12,800.00$ \\
\hline $\begin{array}{l}\text { Especialista en } \\
\text { Inversión Pública }\end{array}$ & $5,000.00$ & $60,000.00$ & & $12,800.00$ \\
\hline $\begin{array}{l}\text { Especialista en Gestión General de } \\
\text { Recursos Públicos }\end{array}$ & $5,000.00$ & $60,000.00$ & & $12,800.00$ \\
\hline Implantador SIAF & $5,000.00$ & $60,000.00$ & & $12,800.00$ \\
\hline Implantador SIAF & $5,000.00$ & $60,000.00$ & & $12,800.00$ \\
\hline Total Anual & $\mathbf{7 9 2 , 0 0 0 . 0 0}$ & $\mathbf{9 2 , 9 9 7 . 6 3}$ & $\mathbf{1 6 6 , 4 0 0 . 0 0}$ \\
\hline Presupuesto anual ejecutado del CONECTAMEF Puno & & $\mathbf{1 , 0 5 1 , 3 9 7 . 6 3}$ \\
\hline
\end{tabular}

Fuente: Portal de transparencia de la entidad.

Por tanto, el ahorro anual estimado, vendría a ser la diferencia entre el gasto estimado (viáticos y pasajes) que asumían las entidades sin CONECTAMEF y el presupuesto anual ejecutado por el CONECTAMEF, es decir:

$$
\mathrm{S} / 4,042,430.00-\mathrm{S} / 1,051,397.63=\mathrm{S} / \mathbf{2 , 9 9 1 , 0 3 2 . 3 7}
$$




\section{Journal of the Academy $|65|$}

De esta manera, se advierte que existe una relación significativa entre el cumplimiento de los servicios brindados por el CONECTAMEF y el ahorro anual de las municipalidades de la región de Puno.

Ahora, respecto a la satisfacción de los servicios brindados por el CONECTAMEF, tal y conforme indicó Muller (2003), Zuleta et al., (2014), Serna (1999) y Olvera et al., (2009), refiere a la acción para atender la necesidad de un cliente que en el trabajo de investigación refiere a las municipalidades distritales de la región de Puno (Ministerio de Comercio Exterior y Turismo, 2002). Asimismo, existen teorías sobre calidad, como las planteadas por Cantú (1997) y Duque (2005), las mismas que se asocian con el objetivo específico 2 del trabajo de investigación.

Al respecto, Cacsire (2012), en su investigación sobre los: Efectos del Proceso de Descentralización Administrativa y Presupuestal en la Gestión de las Direcciones Regionales del Gobierno Regional Puno - Caso DIRCETUR concluyó que, los indicadores de eficacia de la DIRCETUR Puno (2004-2007), respecto al Presupuesto Institucional de Apertura - PIA, presenta un valor promedio de una ejecución del gasto anual de 0.93 y respecto al Presupuesto Institucional Modificado - PIM, la ejecución del gasto anual presenta un valor de 0.96 , siendo ambos indicadores menor que 1 , lo que caracteriza un indicador DEFICIENTE; estableciendo de esta manera que en algunas unidades ejecutoras del Gobierno Regional Puno, hace falta que la atención en la capacitación y asistencia técnica, información que debería ser tomada en cuenta por el CONECTAMEF Puno.

\section{CONCLUSIONES}

Los niveles de cumplimiento de los servicios brindados por el CONECAMEF Puno en el periodo 2017 tienen los siguientes resultados; 4 provincias (30.77\%), indican que el nivel de cumplimiento es "Indiferente", 8 (61.54\%) un nivel "Satisfactorio" y 1 (7.69\%) un nivel "Muy Satisfactorio"; respecto a los niveles de calidad de gasto público de las municipalidades distritales analizadas, el $15.39 \%$ se encuentran en la escala "Mínimo Necesario", el 76.92\% en la escala "Bueno" y el 7.69\% tiene un nivel "Muy Bueno"; al realizar la prueba de hipótesis Chi-Cuadrado " $\mathrm{X}$ " el resultado es: 0,001 demostrando que si existe relación, y según coeficiente de Cramer esta es de un 83.7\%; concluyendo que existe una relación significativa entre el nivel de cumplimiento de los servicios brindados por el CONECTAMEF y el nivel de calidad del gasto público de las municipalidades distritales de la región de Puno

Respecto al ahorro anual estimado anual de las municipalidades de la región de Puno, según los resultados obtenidos en el trabajo de investigación, éste es considerable y viene a ser la diferencia entre el gasto estimado (viáticos y pasajes) que asumían las entidades sin CONECTAMEF y el presupuesto anual ejecutado por el CONECTAMEF, es decir:

$$
\mathrm{S} / 4,042,430.00-\mathrm{S} / 1,051,397.63=\mathrm{S} / 2,991,032.37
$$

Concluyendo que existe una relación significativa entre el nivel de cumplimiento de los servicios brindados por el CONECTAMEF y el ahorro anual de las municipalidades de la región de Puno.

Finalmente, los resultados del trabajo de investigación, evidencian que el CONECTAMEF, es una propuesta de gestión contrastada con la realidad, con resultados muy positivos, que en este caso son reflejados en la mejora de la calidad del gasto público y el ahorro anual de las municipalidades, asimismo, la información proporcionada tiene alcance y servirá como medio de consulta para las instituciones involucradas en el tema. 


\section{Journal of the Academy $|66|$}

\section{REFERENCIAS BIBLIOGRÁFICAS}

Apaza, M. (2014) El Impacto del Programa JUNTOS y de las remesas en el mercado de crédito rural el caso Puno- Perú. Tesis de doctorado. Perú: Universidad Nacional del Altiplano, Puno.

Cacsire, G. (2012). Efectos del proceso de descentralización administrativa y presupuestal en la gestión de las direcciones regionales del Gobierno Regional Puno - Caso DIRCETUR. Tesis de maestría. Perú: Universidad Nacional del Altiplano, Puno.

Cantú, H. (1997). Desarrollo de una cultura de calidad. México: McGraw Hill.

Catacora, V. (2017). Evaluación del impacto del Proyecto: Fortalecimiento de capacidades en el engorde de vacunos de las comunidades del Lago del distrito de Acora 2009-2016. Tesis de maestría. Perú: Universidad Nacional del Altiplano, Puno.

Choquehuanca, H. (2007). Valoración de beneficios para proyectos de drenaje de aguas pluviales caso: ciudad de Juliaca. Tesis de maestría. Perú: Universidad Nacional del Altiplano, Puno.

Duque, E. (2005). Calidad del sevicio y sus modelos bueno. https://doi.org/ 10.1203/PDR.0b013e318213565e.

Espezúa, G. (2014). Política social y Seguridad alimentaria en Puno descentralización de los Programas Sociales (Caso: El Programa del Vaso de Leche). Tesis de maestría. Perú: Universidad Nacional del Altiplano, Puno.

Gutiérrez, R. (2018). Factores críticos que influyen en la gestión de obras públicas y su impacto en el desarrollo de la región Puno. Tesis de doctorado. Perú: Universidad Nacional del Altiplano, Puno.

Hernández, S. R., Fernández, C. C. y Baptista, L. P. (2010). Metodología de la Investigación (5a ed.). México, D.F: Mc Graw Hill Interamericana.

Maquera, N (2009). Análisis de la descentralización de los Programas Sociales en Ilave, con Énfasis en los Comedores Populares. Tesis de maestría. Perú: Universidad Nacional del Altiplano, Puno.

Ministerio de Economía y Finanzas, (2018). Recuperado de https://www.mef.gob.pe/es/ presupuestopor-resultados/ique-es-ppr

Ministerio de Comercio Exterior y Turismo, (2002). Recuperado de https://www.mincetur .gob.pe/LEY_Nro_27783.pdf )

Muller, E. (2003). Cultura de calidad de servicio. México: Trillas.

Olvera, I. y Scherer, A. (2009). El Cliente y la Calidad en el Servicio (1era. ed.). México: Impresora Publimex, S. A. de C. V.

Serna, H. (1999). Servicio al cliente. Métodos de auditoría y medición. Bogotá, Colombia: 3R Editores.

Zuleta, D., Clemenza, D., y Araujo, R. (2014). Calidad de servicio percibida por los usuarios de los programas de postgrado en la gerencia de la facultad de ciencias económicas y sociales, CV(1), 16. 